\title{
Proton stereotactic body radiation therapy for non-small cell lung cancer
}

\author{
Tejan Diwanji ${ }^{1}$, Amit Sawant ${ }^{2,3}$, Terence T. Sio ${ }^{4}$, Nirav V. Patel ${ }^{1}$, Pranshu Mohindra ${ }^{2,3}$ \\ ${ }^{1}$ Department of Radiation Oncology, University of Miami Miller School of Medicine, Sylvester Comprehensive Cancer Center, Miami, FL, USA; \\ ${ }^{2}$ Department of Radiation Oncology, University of Maryland School of Medicine, Baltimore, MD, USA; ${ }^{3}$ Maryland Proton Treatment Center \\ Baltimore, MD, USA; ${ }^{4}$ Department of Radiation Oncology, Mayo Clinic, Phoenix, Arizona, USA \\ Correspondence to: Pranshu Mohindra, MD, MBBS. Associate Professor of Radiation Oncology, University of Maryland School of Medicine, 22 S. \\ Greene St., Rm GGJ35, Baltimore, MD 21201, USA. Email: pranshumohindra@gmail.com. \\ Comment on: Li Y, Dykstra M, Best TD, et al. Differential inflammatory response dynamics in normal lung following stereotactic body radiation \\ therapy with protons versus photons. Radiother Oncol 2019;136:169-75.
}

Submitted Mar 29, 2020. Accepted for publication Apr 14, 2020.

doi: $10.21037 /$ atm-20-2975

View this article at: http://dx.doi.org/10.21037/atm-20-2975

Over the last decade, stereotactic body radiation therapy (SBRT) has rapidly become the new standard of care for the management of inoperable early-stage non-small cell lung cancer (NSCLC) $(1,2)$. In parallel, there has been growing interest in utilizing proton beam therapy (PBT) as means to further enhance the therapeutic ratio by reducing the dose to surrounding normal tissue (3). In particular, there has been a concerted effort to develop this technology for thoracic malignancies (4-8). Thoracic PBT is more prone to the inherent uncertainties of thoracic radiotherapy due to tissue heterogeneity along the beam path, target/organ motion, and its consequent interplay effect $(2,5,9-12)$. Despite these potential limitations, dosimetric studies comparing SBRT delivered with protons versus photons showed substantial benefits in reducing heart and lung doses particular for larger and central tumors (13-17). While similar dosimetric data suggested benefit in locally advanced setting corresponding clinical data thus far have not conclusively demonstrated significant advantages for PBT over photon-based intensity-modulated radiation therapy (IMRT) $(18,19)$. Analogous clinical data for PBT in early stage lung cancer is even more limited.

The juxtaposition of the dosimetric advantage and uncertainty regarding true clinical benefit in the locally advanced NSCLC setting suggest the presence of unaccounted factors. One consideration would be a differential biological effect of protons and photons in normal tissues. This hypothesis was explored by Shusharina et al. as they studied imaging surrogates of normal lung toxicity in patients from the randomized proton $v s$. photon clinical trial (20). The authors utilized post-treatment ${ }^{18}$ F-FDG PET-CT images, which they co-registered to pretreatment planning CT scans to correlate dose to normaltissue and ${ }^{18} \mathrm{~F}$-FDG uptake. Despite wide variability in delivered normal tissue doses, the relationship between tracer uptake and dose did not differ between protons and photons (20). In a separate analysis of data from the same clinical trial, they also demonstrated that post-treatment lung ${ }^{18} \mathrm{~F}$-FDG uptake correlated well with the severity of radiation pneumonitis within 6 months of completing chemoradiation (regardless of radiation treatment modality) (21). Another approach to compute lung inflammation through ${ }^{18} \mathrm{~F}$-FDG PET-CT involves quantification of pulmonary parenchymal glycolysis (PG), measured as the summation of uptake values throughout the lung fields. This technique was utilized in a small retrospective study from the University of Pennsylvania (22), in which the authors compared the levels of inflammation in 9 patients treated with photons vs. 9 patients treated with protons. Contrary to the prior study, the authors found a small change in PG values with photon therapy, but no analogous changes were noted after proton therapy. Furthermore, unlike the prior study, the authors could not correlate the quantitative changes in lung inflammation to radiation pneumonitis.

While the initial exploratory work has been performed in the locally advanced NSCLC setting, corresponding data for SBRT was notably absent. As such, we read with great interest the work by $\mathrm{Li}$ et al. exploring an imaging 
surrogate of differential inflammatory response in normal lung after SBRT with both protons and photons (23). Unlike the locally advanced setting where patients often receive post-treatment PET scans, SBRT patients usually undergo surveillance with serial CT scans alone (24). As such, the authors used a previously validated CT based biomarker of lung damage (25). The authors undertook a single-institution matched-pair retrospective analysis across 46 patients treated at the Massachusetts General Hospital. Notably, 5 patients received both proton and photon treatments and as such were also analyzed as part of an internal control cohort. The authors found that patients treated with proton therapy demonstrated a significant change in lung density during the early time period (defined as $<6$ months), which often subsequently stabilized in the late period. This was different than the effect noted in patients treated with photons where similar early changes were noted, but progressive significant changes were noted at later time points as well. Significantly, more patients in the proton arm had "severe" inflammatory responses on imaging (5 Proton vs. 3 photon patients). Furthermore, among the patients deemed "sensitive", i.e., patients who demonstrated large density changes per unit of radiation dose, the differential density changes between proton and photon-treated patients from the early to late time period was magnified. Similar results were also noted among the patients in the internal control cohort of 5 patients who received both proton and photon treatments. Interestingly, the test for variability in maximum lung response by this technique did not reach statistical significance but did trend toward the proton arm. Secondarily, in an exploratory analysis restricted to areas of the lung receiving low-dose radiation, there was a weak trend toward increased doseresponse for protons versus photons. Finally, the authors also performed a blinded qualitative analysis which did agree well with their quantitative results.

This study has numerous strengths, including the patient selection which was performed via matching pairs across multiple tumor characteristic, demographic, and treatment domains thereby minimizing selection bias as much as reasonably possible. The authors also utilized a welldefined, previously validated methodology for isolating the lung region of interest and quantifying dose and density. Some weaknesses include the retrospective nature and all of its inherent susceptibilities to potential bias. Furthermore, the authors do not specify the uncertainties in their deformable image registration. Given that the follow-up CT scans are rarely done in treatment position often time using breath-hold, and are potentially acquired at a different resolution than the treatment-planning CT, the analyses will be associated with registration-related uncertainties, which may impact the interpretation of this comparative study. Additionally, the treatments were performed with passive scatter technology and dose calculations were based on a pencil-beam model. These are increasingly being replaced by pencil-beam scanning delivery techniques and also with more computationally intensive but reliable Monte-Carlo dose calculation algorithms. As both of these could impact estimates of dose deposition, their analyzed results could be impacted which may make the study less generalizable.

The authors did not report on systemic steroid/and anti-inflammatory medication use in this population or symptomatic implications of these radiological changes, which could certainly confound the data particularly between the early and late phases. An older retrospective experience of 3-5 fraction proton SBRT (42-50 Gy) from the same institution showed grade 1 pneumonitis in $30 \%$ patients, with grade $2 / 3$ event seen only in 1 patient (26). A four-fraction carbon-ion based SBRT series of 79 patients from another Japanese center revealed acute RTOG grade $1 / 2$ events in 1 patient, and with no other grade $3 / 4$ events (27). Late grade 1 respiratory event was noted in $88 \%$ of the patients. In the current series, one-third of the patients showed no distinct or low responses in normal lung, suggesting the other two-third of patients did have radiographic changes. Extrapolating information from both their own and the Japanese experience, only a small proportion of these patients may have experienced highgrade/symptomatic events. As such, the clinical utility of their work would be augmented if the rate of development of radiological changes could be correlated with symptom evolution.

Another factor relates to the inherent differences in planning techniques between proton (SBPT) and photonbased (SBRT) regimens and its impact on outcomes. While multiple dosimetric studies have demonstrated these differences, a randomized phase II trial comparing SBRT $v s$. SBPT for high-risk early stage NSCLC conducted at the host institution in collaboration with MD Anderson Cancer Center showed that the volume of PTV was much larger in the SBPT group (44.4 vs. $17.2 \mathrm{cc}$ ); and the median PTV maximum dose was higher in SBRT group (68.4 vs. 57.3 Gy RBE) (28). Such dosimetric data was not provided in the $\mathrm{Li}$ et al. study. While the randomized study was closed early due to poor accrual, acute grade $1 / 2$ dyspnea and/or cough were seen in 4 of the 10 SBPT patients, versus 0 (none) of 
the 9 SBRT patients. No grade 3 respiratory toxicity was seen, and late lung fibrosis was similar in both cohorts. These differences in planning approaches could impact biological impact between volume effect versus dose-per fraction. Many modern intensity-modulated proton therapy (IMPT)-based centers now have advanced IGRT abilities which raise the possibility of reducing PTV volumes, while still allowing central dose escalation within GTV akin to photon-SBRT - a future research and clinical trial direction. It remains to be seen if similar trend of differing radiological responses as seen the analysis by $\mathrm{Li}$ et al. can be observed in such future studies with improved protonSBRT planning. Selected centers in the United States have also developed linear energy transfer (LET) and relative biological effectiveness (RBE)-based algorithms for robust proton treatment planning (29).

In summary, Li et al. help establish a differential response of both protons and photons in normal lung parenchyma using radiological differences by Hounsfield Unit changes in CT scan per unit change in the SBRT dosing. In the future, such work can be augmented by incorporating pretreatment regional heart and lung function information (such as ventilation-perfusion scan and echocardiogram data) into the post-treatment sensitivity analysis, along with inclusion of clinically relevant end-points. Supporting data on circulatory biomarkers of inflammation could further bolster our understanding of these temporal patterns observed clinically. Incorporating complimentary imaging data including ${ }^{18}$ F-FDG PET-CT, SPECT, PET-MR, or Dynamic Contrast Enhanced Magnetic Resonance Imaging could additionally provide multiparametric assessments of normal tissue dysfunction and potentially strengthen the observed clinical correlation to radiation-induced normal tissue injuries. Future IMPT studies should also focus on refining treatment planning methods which account for breathing-motion related interplay effects; these can be accomplished by improving Monte-Carlo based calculation methods, novel fast computing and artificial intelligence tools, and improved robust motion-based optimization techniques. An understanding of differences in dosimetric parameters and the possible biological effect consequences by IMPT technologies will be critical in being able to understand the full potential of proton-based SBRT which will help in the design of pragmatic clinical trials looking at relevant end-points (30). From a clinical practicality standpoint, based on the data from dosimetric studies and early clinical experiences so far (which are limited), protonbased SBRT may play a key role for higher-risk settings including larger and/or central tumors wherever photonbased SBRT techniques are limited by excess dose to lungs and other centrally located mediastinal structures (e.g., heart, esophagus and spine). Integration of immune modulators with proton therapy taking advantage of any differing biological effects (from better normal tissue sparing or from in-field immune response) will need to be studied further. Using more modern delivery technologies and with better understanding of biology the future of thoracic proton radiotherapy continues to show promise.

\section{Acknowledgments}

Funding: None.

\section{Footnote}

Provenance and Peer Review: This article was commissioned and reviewed by the Section Editor Dr. Hsin-Hua Nien (Attending physician, Department of Radiation Oncology, Cathay General Hospital, Taipei).

Conflicts of Interest: All authors have completed the ICMJE uniform disclosure form (available at http://dx.doi. org/10.21037/atm-20-2975). AS reports grants from Varian Medical Systems, non-financial support from Vision RT Inc., outside the submitted work; TTS reports non-financial support from Novocure, Inc., outside the submitted work. The other authors have no conflicts of interest to declare.

Ethical Statement: The authors are accountable for all aspects of the work in ensuring that questions related to the accuracy or integrity of any part of the work are appropriately investigated and resolved.

Open Access Statement: This is an Open Access article distributed in accordance with the Creative Commons Attribution-NonCommercial-NoDerivs 4.0 International License (CC BY-NC-ND 4.0), which permits the noncommercial replication and distribution of the article with the strict proviso that no changes or edits are made and the original work is properly cited (including links to both the formal publication through the relevant DOI and the license). See: https://creativecommons.org/licenses/by-nc-nd/4.0/.

\section{References}

1. Timmerman R, Paulus R, Galvin J, et al. Stereotactic body 
radiation therapy for inoperable early stage lung cancer. JAMA 2010;303:1070-6.

2. Diwanji TP, Mohindra P, Vyfhuis M, et al. Advances in radiotherapy techniques and delivery for non-small cell lung cancer: benefits of intensity-modulated radiation therapy, proton therapy, and stereotactic body radiation therapy. Transl Lung Cancer Res 2017;6:131-47.

3. Allen AM, Pawlicki T, Dong L, et al. An evidence based review of proton beam therapy: the report of ASTRO's emerging technology committee. Radiother Oncol 2012;103:8-11.

4. Badiyan SN, Molitoris JK, Zhu M, et al. Proton beam therapy for malignant pleural mesothelioma. Transl Lung Cancer Res 2018;7:189-98.

5. Vyfhuis MAL, Onyeuku N, Diwanji T, et al. Advances in proton therapy in lung cancer. Ther Adv Respir Dis 2018;12:1753466618783878.

6. Chuong MD, Larson GL, Hartsell WF, et al. Limited Toxicity After Proton Beam Therapy for Esophageal Cancer: Outcomes From the Proton Collaborative Group. Int J Radiat Oncol Biol Phys 2016;96:E152-3.

7. Willmann J, Rimner A. The expanding role of radiation therapy for thymic malignancies. J Thorac Dis 2018;10:S2555-64.

8. Yu NY, DeWees TA, Liu C, et al. Early Outcomes of Patients With Locally Advanced Non-small Cell Lung Cancer Treated With Intensity-Modulated Proton Therapy Versus Intensity-Modulated Radiation Therapy: The Mayo Clinic Experience. Adv Radiat Oncol 2019. doi: 10.1016/j.adro.2019.08.001.

9. Szeto YZ, Witte MG, van Kranen SR, et al. Effects of anatomical changes on pencil beam scanning proton plans in locally advanced NSCLC patients. Radiother Oncol 2016;120:286-92.

10. Dowdell S, Grassberger C, Sharp G, et al. Fractionated Lung IMPT Treatments: Sensitivity to Setup Uncertainties and Motion Effects Based on Single-Field Homogeneity. Technol Cancer Res Treat 2016;15:689-96.

11. Li H, Zhang X, Li Y, et al. TH-C-BRD-07: Minimizing Dose Uncertainty for Spot Scanning Beam Proton Therapy of Moving Tumor with Optimization of Delivery Sequence. Medical Physics 2014;41:551-2.

12. Tryggestad EJ, Liu W, Pepin MD, et al. Managing treatment-related uncertainties in proton beam radiotherapy for gastrointestinal cancers. J Gastrointest Oncol 2020;11:212-24.

13. Welsh J, Amini A, Ciura K, et al. Evaluating proton stereotactic body radiotherapy to reduce chest wall dose in the treatment of lung cancer. Med Dosim 2013;38:442-7.

14. Hoppe BS, Huh S, Flampouri S, et al. Double-scattered proton-based stereotactic body radiotherapy for stage I lung cancer: a dosimetric comparison with photonbased stereotactic body radiotherapy. Radiother Oncol 2010;97:425-30.

15. Nichols RC, Huh SH, Hoppe BS, et al. Protons safely allow coverage of high-risk nodes for patients with regionally advanced non-small-cell lung cancer. Technol Cancer Res Treat 2011;10:317-22.

16. Register SP, Zhang X, Mohan R, et al. Proton stereotactic body radiation therapy for clinically challenging cases of centrally and superiorly located stage I non-small-cell lung cancer. Int J Radiat Oncol Biol Phys 2011;80:1015-22.

17. Seco J, Panahandeh HR, Westover K, et al. Treatment of non-small cell lung cancer patients with proton beam-based stereotactic body radiotherapy: dosimetric comparison with photon plans highlights importance of range uncertainty. Int J Radiat Oncol Biol Phys 2012;83:354-61.

18. Liao Z, Lee JJ, Komaki R, et al. Bayesian Adaptive Randomization Trial of Passive Scattering Proton Therapy and Intensity-Modulated Photon Radiotherapy for Locally Advanced Non-Small-Cell Lung Cancer. J Clin Oncol 2018;36:1813-22.

19. Higgins KA, O'Connell K, Liu Y, et al. National Cancer Database Analysis of Proton Versus Photon Radiation Therapy in Non-Small Cell Lung Cancer. Int J Radiat Oncol Biol Phys 2017;97:128-37.

20. Shusharina N, Liao Z, Mohan R, et al. Differences in lung injury after IMRT or proton therapy assessed by (18)FDG PET imaging. Radiother Oncol 2018;128:147-53.

21. Yue J, McKeever M, Sio TT, et al. Association of lung fluorodeoxyglucose uptake with radiation pneumonitis after concurrent chemoradiation for non-small cell lung cancer. Clin Transl Radiat Oncol 2017;4:1-7.

22. Rice SR, Saboury B, Houshmand S, et al. Quantification of global lung inflammation using volumetric 18F-FDG PET/CT parameters in locally advanced non-smallcell lung cancer patients treated with concurrent chemoradiotherapy: a comparison of photon and proton radiation therapy. Nucl Med Commun 2019;40:618-25.

23. Li Y, Dykstra M, Best TD, et al. Differential inflammatory response dynamics in normal lung following stereotactic body radiation therapy with protons versus photons. Radiother Oncol 2019;136:169-75.

24. National Comprehensive Cancer Network. Non Small Cell Lung Cancer (Version 3.2020). 2020. Available online: 
https://www.nccn.org/professionals/physician_gls/pdf/ nscl.pdf. Accessed 3/26/2020.

25. Veiga C, Landau D, Devaraj A, et al. Novel CT-Based Objective Imaging Biomarkers of Long-Term RadiationInduced Lung Damage. Int J Radiat Oncol Biol Phys 2018;102:1287-98.

26. Westover KD, Seco J, Adams JA, et al. Proton SBRT for medically inoperable stage I NSCLC. J Thorac Oncol 2012;7:1021-5.

27. Miyamoto T, Baba M, Sugane T, et al. Carbon ion radiotherapy for stage I non-small cell lung cancer using a regimen of four fractions during 1 week. J Thorac Oncol 2007;2:916-26.

Cite this article as: Diwanji T, Sawant A, Sio TT, Patel NV, Mohindra P. Proton stereotactic body radiation therapy for non-small cell lung cancer. Ann Transl Med 2020;8(18):1198. doi: 10.21037/atm-20-2975
28. Nantavithya C, Gomez DR, Wei X, et al. Phase 2 Study of Stereotactic Body Radiation Therapy and Stereotactic Body Proton Therapy for High-Risk, Medically Inoperable, Early-Stage Non-Small Cell Lung Cancer. Int J Radiat Oncol Biol Phys 2018;101:558-63.

29. An Y, Shan J, Patel SH, et al. Robust intensity-modulated proton therapy to reduce high linear energy transfer in organs at risk. Medical physics 2017;44:6138-47.

30. Gentile MS, Yeap BY, Paganetti H, et al. Brainstem Injury in Pediatric Patients With Posterior Fossa Tumors Treated With Proton Beam Therapy and Associated Dosimetric Factors. Int J Radiat Oncol Biol Phys 2018;100:719-29. 